\title{
Combined Therapy With Functional Electrical Stimulation and Standing Frame in Stroke Patients
}

\author{
Joung Bok Lee, $\mathrm{MD}^{1,2}$, Sang Beom Kim, $\mathrm{MD}^{1,2}$, Kyeong Woo Lee, $\mathrm{MD}^{1,2}$, \\ Jong Hwa Lee, $\mathrm{MD}^{1,2}$, Jin Gee Park, $\mathrm{MD}^{1,2}$, Sook Joung Lee, $\mathrm{MD}^{3}$
}

\begin{abstract}
${ }^{1}$ Department of Physical Medicine and Rehabilitation, Dong-A University College of Medicine, Busan; ${ }^{2}$ Regional Cardiocerebrovascular Center, Dong-A University Medical Center, Busan; ${ }^{3}$ Department of Physical Medicine and Rehabilitation, Daejeon St. Mary's Hospital, College of Medicine, The Catholic University of Korea, Seoul, Korea
\end{abstract}

Objective To investigate the effects of combination functional electrical stimulation (FES) and standing frame training on standing balance in stroke patients.

Methods Patients who had hemiparesis and postural instability after stroke were randomly assigned to one of the two groups; study group underwent FES on the quadriceps and tibialis anterior muscle simultaneously with standing balance training. The control group received standing frame training and FES separately. Both the groups received their respective therapies for 3 weeks. Stability index in Biodex Balance master system, Berg Balance Scale (BBS), manual muscle test, the Korean version of Modified Barthel Index, and Korean version of MiniMental State Examination were used to evaluate the effects of the treatment.

Results In total, 30 patients were recruited to the study group and 30 to the control group. Three weeks after treatment, both the groups showed improvement in postural stability scores and physical and cognitive functions. When changes in postural stability were compared between the groups, the study group showed more significant improvement than the control group with regards to the scores of BBS and the stability indices.

Conclusion In this study, we found the therapeutic effectiveness of combined therapy of FES and standing frame in subacute stroke patients. The presented protocol is proposed as time-saving and can be applied easily in the clinical setting. Thus, the proposed combined therapy could be a useful method for improving standing balance in subacute stroke patients.

Keywords Stroke, Standing frame, Functional electrical stimulation, Standing balance, Rehabilitation

Received June 1, 2018; Accepted September 27, 2018

Corresponding author: Sook Joung Lee

Department of Physical Medicine and Rehabilitation, Deajeon St. Mary's Hospital, College of Medicine, The Catholic University of Korea, 64 Daeheung-ro, Jung-gu, Daejeon 34943, Korea. Tel: +82-42-220-9650, Fax: +82-42-220-9059, E-mail: 1sj995414@hanmail.net

ORCID: Joung Bok Lee (http://orcid.org/0000-0001-7741-1092); Sang Beom Kim (http://orcid.org/0000-0002-5622-5933); Kyeong Woo Lee (http:// orcid.org/0000-0001-8785-5535); Jong Hwa Lee (http://orcid.org/0000-0003-2489-358X); Jin Gee Park (http://orcid.org/0000-0003-2672-1618); Sook Joung Lee (http://orcid.org/0000-0002-6894-445X).

(c) This is an open-access article distributed under the terms of the Creative Commons Attribution Non-Commercial License (http://creativecommons.org/ licenses/by-nc/4.0) which permits unrestricted noncommercial use, distribution, and reproduction in any medium, provided the original work is properly cited. Copyright $\odot 2019$ by Korean Academy of Rehabilitation Medicine 


\section{INTRODUCTION}

Hemiplegia occurs in several patients after a stroke $[1,2]$ and results in a decline in the patient's standing ability $[3,4]$. Patients with poor standing ability are prone to gait disturbances that lead to falling and impairment in activities of daily living, ultimately resulting in a deteriorated quality of life $[5,6]$. Thus, improvement in standing ability is an important part of rehabilitation therapy for stroke patients $[7,8]$.

In order to improve standing ability, various treatments, such as exercise therapy, drug therapy, and electric stimulation therapy have been employed [9-11]. One of the various exercise therapy methods used for improving standing ability is the standing frame therapy. Previous studies have reported the effectiveness of standing frame training in improving the standing ability of stroke patients $[12,13]$.

However, in the actual practice of standing frame training, patients tend to stand with the weight more on the healthy side without weight bearing on the hemiplegic side $[14,15]$, resulting in dissatisfactory exercise effect on the hemiplegic side $[16,17]$. Therefore, to achieve effective improvement in the standing ability through rehabilitation therapy, patients should be encouraged to perform weight bearing on the hemiplegic side and not on the healthy side $[18,19]$. Various methods have been investigated to resolve this problem.

In the present study, functional electrical stimulation (FES) of the leg on the hemiplegic side was investigated. FES is a widely used treatment method with proven effective rehabilitation therapy for improving the standing ability of stroke patients by improving muscle strengthening on their hemiplegic leg $[20,21]$. Furthermore, it was found to help sensory recovery and body perception on the hemiplegic side through a sensory feedback mechanism [22,23].

We hypothesized that if patients used the standing frame simultaneously with FES, FES would increase the strength of the hemiparesis and sensory feedback to induce weight bearing on the hemiplegic side during the standing frame training.

The purpose of this study was to investigate the effect of combination therapy involving standing frame training and FES in stroke patients, thereby proving the above hypothesis.

\section{MATERIALS AND METHODS}

\section{Subjects}

This study was conducted on subacute stroke patients referred to the Regional Cardiocerebrovascular Center of our hospital from January 2015 to December 2016. The patients were selected based on the physical examination and the inclusion criterion was: the ability to maintain the sitting balance with the ability to control their neck and trunk, but unable to stand independently for more than a minute, thus requiring standing balance training. In addition, patients in whom the motor power grade of the leg on the hemiplegic side was $\leq 3$ as per the manual muscle test (MMT) were enrolled in this study.

Patients who were unable to cooperate due to deteriorated cognitive function and those with a past history of stroke, a musculoskeletal impairment that could affect posture imbalance, or standing balance disturbance due to ataxia were excluded.

All patients who met the eligibility criteria were randomly assigned to the study group (treated with combination therapy of FES and standing frame training simultaneously) or to the control group (treated with FES and standing frame training separately) using a computergenerated randomization table according to the random permuted blocks of two.

\section{Intervention protocol}

In the study group, FES therapy was applied to the quadriceps femoris muscle and the anterior tibialis on the hemiplegic side while the standing frame training was performed. In the control group, standing frame training and FES on the hemiplegic side were performed separately (Fig. 1). The intensity and frequency of FES therapy were set within the ranges of 20-30 $\mathrm{mA}$ and 30-40 $\mathrm{Hz}$, respectively, according to the personal characteristics of each patient, at which, the contractions of the quadriceps femoris muscle and anterior tibialis muscle could be sensed. The pads for FES therapy were attached on the motor point of the quadriceps femoris muscle that was located at a region $5 \mathrm{~cm}$ above the patella along the median vertical line of the iliac crest and on the motor point of the anterior tibialis muscle that was located at an intersection of the $1 / 3$ point between the knee and ankle and the outer boundary of the tibia. In the study group, standing frame training and FES were applied simultane- 

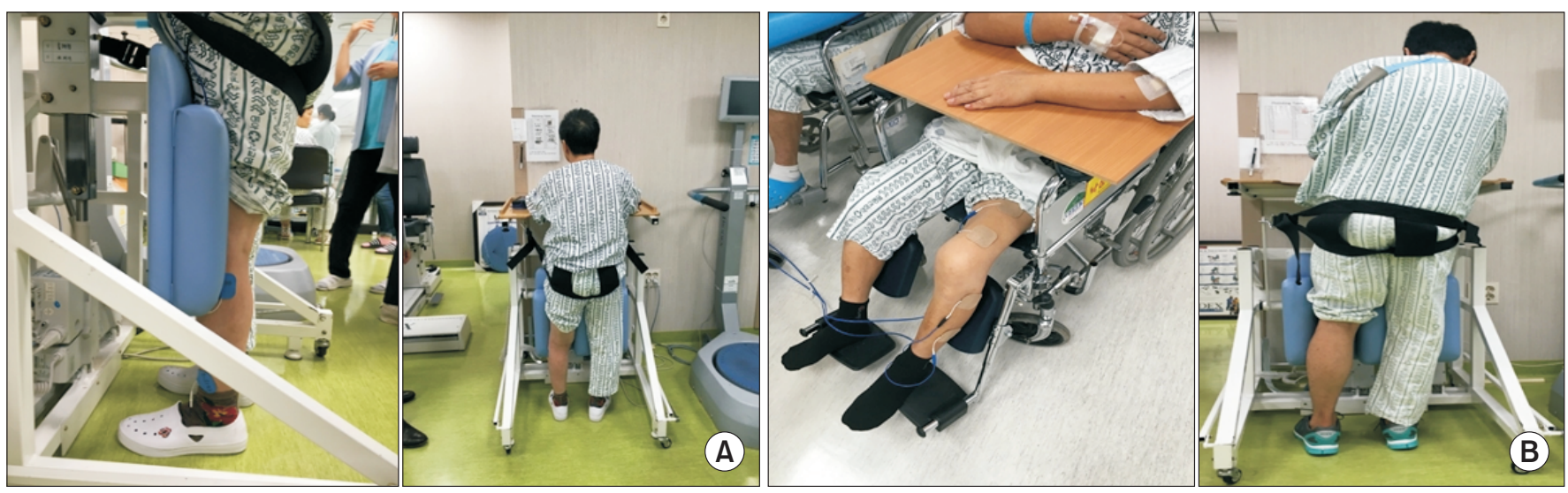

Fig. 1. (A) The study group received combination treatment (standing frame training and FES simultaneously) and (B) the control group received conventional treatment (standing frame training without FES and FES separately). FES, functional electrical stimulation.

ously for 20 minutes per session, twice a day, making a total of 40 minutes (40 minutes per day). In the control group, standing frame training and FES were applied separately for 20 minutes per session, twice a day, making a total of 80 minutes ( 80 minutes per day). A total of 30 treatment sessions were conducted for each patient 5 days a week for 3 weeks.

During the standing frame exercise, the therapist was asked to lift the patient's leg on the non-hemiplegia side or to give a verbal cue to induce weight bearing on the hemiplegia-side.

The groups received conventional rehabilitation treatment for standing balance training by physical therapists and the conventional therapy for 30 minutes per session twice a day, 5 days a week for 3 weeks, for 30 times in total.

\section{Evaluation}

Standing stability determined using posturography and Berg Balance Scale (BBS) were used to assess standing stability and balance. To evaluate the patient's physical and cognitive abilities, the MMT, the Korean version of the Modified Barthel Index (K-MBI), and the Korean version of the Mini-Mental State Examination (K-MMSE) were performed. In addition, the MMT was used to evaluate the leg muscle strength. For basic information about stroke patients, their age, sex, and stroke lesion were investigated.

The BBS was used for the comprehensive evaluation of the patients' sitting balance, standing balance, and walking ability. BBS is a clinical measure of balance consisting of 14 progressively challenging tasks. Each task is graded on a 5-point ordinal scale, and the score obtained is summed to obtain a possible total score ranging from 0 to 56. Scoring is based on the ability to meet a certain time or distance requirements and the ability to perform the tasks independently $[24,25]$.

In addition, posturography-based evaluation was performed using Balance master system (Biodex Medical System, New York, NY, USA). The posturography test was conducted as follows: the patient ascended on a test instrument and maintained the standing balance for 10 seconds; thereafter, the balance master machine measured the degree of balance during the weight load for those 10 seconds, measured as the stability index value. The stability index consists of the anterior-posterior stability index that measures the degree of the anterior and posterior weight load of the patient, the medial-lateral stability index that measures the bilateral weight load of the patient, and the overall stability index that indicates the patient's overall sense of balance. Each value ranged from a minimum of 0 to a maximum of 14 points; higher the score, the more scattered the patient's weight load, indicating poor standing ability [26]. Posturography could not be measured for all patients because of their inability to stand independently before the initiation of therapy; therefore, patients who could not be examined were given the initial stability index as their maximum score.

All the evaluations were measured before and after 3 weeks of the rehabilitation program. All the tests were performed under the same conditions by two rehabilita- 
tion specialists.

During the entire rehabilitation training period, no changes in the medication were made, and no other treatments that could affect the standing ability were followed.

Approval for the study was obtained from the Institutional Review Board of Dong-A University Medical Center (No. 18-068), and written consent was received from all the enrolled patients.

\section{Statistical analysis}

The SPSS version 21.0 for Windows (IBM, Armonk, NY, USA) was used for statistical analysis. Comparisons between the study group and the control group were statistically processed through Mann-Whitney U-test. Also, an evaluation of all the indicators, including various indicators to determine the degree of standing balance was conducted using the Wilcoxon signed-rank test. Statistical significance was defined for cases where the $p$-value was below 0.05 .

\section{RESULTS}

During the study period, 190 stroke patients were admitted to our department. In total, 116 patients were excluded from the study and 74 patients who could not stand due to stroke were recruited. From these, 35 were randomly assigned to the study group and 39 were assigned to the control group. Fourteen patients dropped out due to early discharge from the hospital or decline in their medical condition. Finally, 60 patients (30 in the study group and 30 in the control group) who completed all the treatment sessions were investigated (Fig. 2). The study group consisted of 17 males and 13 females, and the mean age of the patients was 67.2 years. In the study group, 17 patients had an ischemic stroke, while 13 had a hemorrhagic stroke. The control group consisted of 16 males and 14 females, and the mean age of the patients was 68.5 years. Eighteen patients had an ischemic stroke, while 12 had a hemorrhagic stroke. At the time of initial evaluation, 6 patients in the study group and 8 patients in the control group could not be examined. There was no significant difference between the groups regarding the initial values of the baseline characteristics (Table 1). In addition, there was no significant difference in the initial postural stability index between the groups (Table 2).

After 3 weeks of standing balance treatment, significant improvements in the values of the BBS, the stability indices, the MMT of the hip muscle, K-MBI, and K-MMSE were observed in both the groups compared to the values measured before the treatment (Table 3).

A statistically significant difference in the BBS score

116 excluded:

9 had a poor medical condition

12 had ataxia

17 had cognitive impairment (MMSE<18)

22 had a previous stroke history

25 had musculoskeletal disability

31 did not have standing difficulty

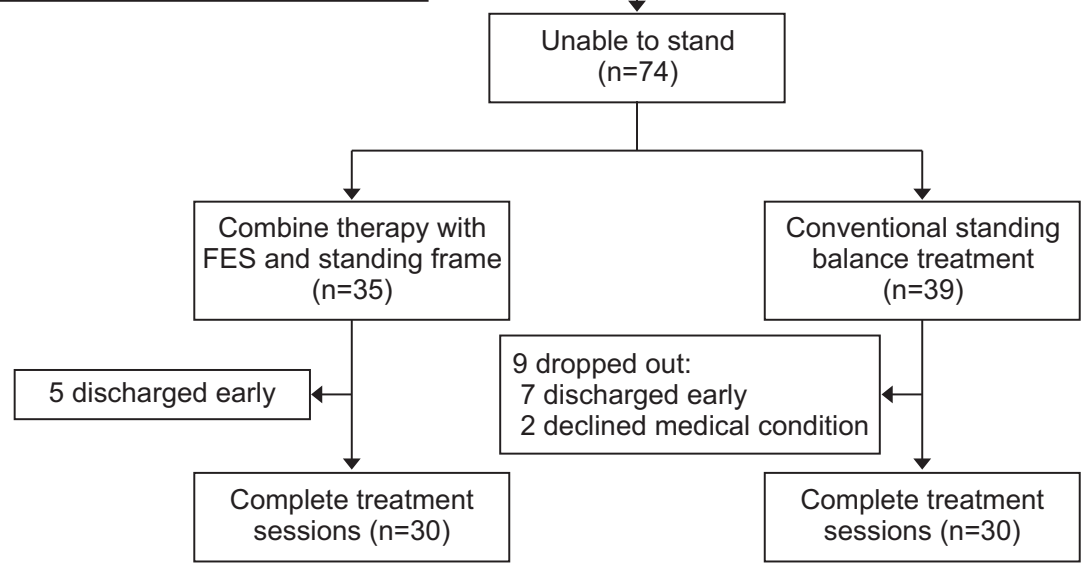

Fig. 2. Flowchart for enrollment of the subjects. MMSE, Mini-Mental State Examination; FES, functional electrical stimulation. 
and the stability indices was noted when the therapeutic effect was compared between the two groups. However, no statistically significant difference was observed in the results of the MMT, K-MBI, and K-MMSE (Table 4).

\section{DISCUSSION}

In stroke patients, the standing ability is an essential skill fundamental to gait and plays an important role in enabling patients to conduct their daily activities independently. In many stroke patients, standing ability declines due to hemiplegia, resulting in a reduced ability to perform activities of daily living. Therefore, rehabilitation therapy that helps stroke patients to improve their standing ability has become an essential part of rehabilitation. Consequently, various rehabilitation therapies are being conducted for effective improvement in the standing ability of stroke patients [27].

Table 1. Baseline demographic characteristics of patients

\begin{tabular}{|cccc|}
\hline Characteristic & Study group $(\mathbf{n = 3 0})$ & Control group $(\mathbf{n = 3 0})$ & p-value $^{\mathbf{a})}$ \\
\hline Age (yr) & $67.25 \pm 15.01$ & $68.50 \pm 15.80$ & 0.915 \\
\hline Sex & $17(57)$ & & 0.850 \\
\hline Male & $13(43)$ & $16(53)$ & \\
\hline Female & & & \\
\hline Stroke & 16 & $14(47)$ & \\
\hline Right & 14 & 13 & \\
\hline Left & 17 & 18 & 0.825 \\
\hline Type of stroke & 13 & 12 & \\
\hline Ischemic & $24.5 \pm 17.4$ & $26.3 \pm 15.4$ & 0.765 \\
\hline Hemorrhagic & & & 0.855 \\
\hline Duration (day) & $1.75 \pm 0.50$ & $1.85 \pm 0.85$ & 0.630 \\
\hline MMT & $1.33 \pm 0.50$ & $1.35 \pm 0.50$ & 0.350 \\
\hline Hip & $1.25 \pm 0.80$ & $1.13 \pm 0.35$ & 0.451 \\
\hline Knee & $17.50 \pm 11.55$ & $21.50 \pm 14.46$ & \\
\hline Ankle & $10.75 \pm 11.53$ & $12.55 \pm 12.55$ & \\
\hline K-MBI & & & \\
\hline K-MMSE & & & \\
\hline
\end{tabular}

Values are numbers or mean \pm standard deviation or number (\%).

MMT, manual muscle test; K-MBI, Korean version of Modified Barthel Index; K-MMSE, Korean version of Mini-Mental State Examination.

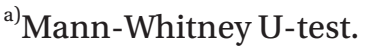

Table 2. Baseline postural stability of patients

\begin{tabular}{|c|c|c|c|}
\hline Characteristic & Study group $(n=30)$ & Control group $(n=30)$ & p-value ${ }^{c)}$ \\
\hline BBS & $5.58 \pm 1.11$ & $5.75 \pm 2.50$ & 0.770 \\
\hline \multicolumn{4}{|l|}{ Stability index } \\
\hline Overall & $12.20 \pm 3.43^{\mathrm{a})}$ & $11.25 \pm 2.55^{\mathrm{b})}$ & 0.820 \\
\hline AP & $9.65 \pm 4.45^{\mathrm{a})}$ & $9.33 \pm 2.05^{\mathrm{b})}$ & 0.866 \\
\hline ML & $6.70 \pm 3.28^{\mathrm{a})}$ & $6.85 \pm 3.51^{\mathrm{b})}$ & 0.365 \\
\hline
\end{tabular}

Values are numbers or mean \pm standard deviation.

BBS, Berg Balance Scale; AP, anterior-posterior; ML, medial-lateral.

${ }^{\text {a) }}$ Since there was a patient who could not be examined at the initial period, only 24 patients could be tested.

${ }^{\text {b) }}$ Since there was a patient who could not be examined at the initial period, only 22 patients could be tested.

${ }^{c)}$ Mann-Whitney U-test. 
Table 3. Changes in postural stability and functional status after treatment

\begin{tabular}{|c|c|c|c|c|c|c|}
\hline & \multicolumn{3}{|c|}{ Study group $(n=30)$} & \multicolumn{3}{|c|}{ Control group $(n=30)$} \\
\hline & Pre & Post & p-value & Pre & Post & p-value \\
\hline BBS & & $17.75 \pm 5.75$ & $0.005^{*}$ & $5.75 \pm 2.50$ & $12.85 \pm 5.35$ & $0.012^{*}$ \\
\hline \multicolumn{7}{|c|}{ Stability index } \\
\hline Overall & $12.20 \pm 3.43^{\mathrm{a})}$ & $6.40 \pm 4.29$ & $0.012^{*}$ & $11.25 \pm 2.55^{\mathrm{b})}$ & $8.20 \pm 4.55$ & $0.035^{*}$ \\
\hline AP & $9.65 \pm 4.45^{\mathrm{a})}$ & $4.20 \pm 3.55$ & $0.018^{*}$ & $9.33 \pm 2.05^{\mathrm{b})}$ & $6.35 \pm 4.22$ & $0.043^{*}$ \\
\hline ML & $6.70 \pm 3.28^{\mathrm{a})}$ & $3.38 \pm 2.55$ & $0.024^{*}$ & $6.85 \pm 3.51^{\mathrm{b})}$ & $5.55 \pm 3.45$ & $0.045^{*}$ \\
\hline \multicolumn{7}{|l|}{ MMT } \\
\hline Hip & $1.75 \pm 0.50$ & $2.25 \pm 0.55$ & $0.012^{*}$ & $1.85 \pm 0.85$ & $2.15 \pm 0.75$ & $0.025^{*}$ \\
\hline Knee & $1.33 \pm 0.50$ & $1.90 \pm 0.75$ & 0.051 & $1.35 \pm 0.50$ & $1.80 \pm 0.82$ & 0.339 \\
\hline Ankle & $1.25 \pm 0.80$ & $1.35 \pm 0.72$ & 0.150 & $1.13 \pm 0.35$ & $1.25 \pm 0.55$ & 0.540 \\
\hline K-MBI & $17.50 \pm 11.55$ & $35.25 \pm 17.45$ & $0.025^{*}$ & $21.50 \pm 14.46$ & $34.75 \pm 19.80$ & $0.030^{*}$ \\
\hline K-MMSE & $10.75 \pm 11.53$ & $16.55 \pm 12.35$ & $0.033^{*}$ & $12.55 \pm 12.55$ & $17.50 \pm 10.95$ & $0.035^{*}$ \\
\hline
\end{tabular}

Values are numbers or mean \pm standard deviation.

BBS, Berg Balance Scale; AP, anterior-posterior; ML, medial-lateral; MMT, manual muscle test; K-MBI, Korean version of Modified Barthel Index; K-MMSE, Korean version of Mini-Mental State Examination.

${ }^{\text {a) }}$ Since there was a patient who could not be examined at the initial period, only 24 patients could be tested.

${ }^{b}$ Since there was a patient who could not be examined at the initial period, only 22 patients could be tested.

* $\mathrm{p}<0.05$ by Mann-Whitney U-test.

Table 4. Comparison of changes between the two groups

\begin{tabular}{lrrr}
\hline & $\begin{array}{c}\text { Study group } \\
(\mathbf{n}=\mathbf{3 0})\end{array}$ & $\begin{array}{c}\text { Control group } \\
(\mathbf{n}=\mathbf{3 0})\end{array}$ & p-value \\
\hline$\Delta$ BBS & $12.17 \pm 4.35$ & $7.10 \pm 3.26$ & $0.043^{*}$ \\
$\Delta$ Stability index & & & \\
\hline Overall & $-4.55 \pm 3.20$ & $-2.35 \pm 2.25$ & $0.045^{*}$ \\
AP & $-4.25 \pm 2.33$ & $-1.70 \pm 2.35$ & $0.042^{*}$ \\
ML & $-3.15 \pm 1.53$ & $-1.40 \pm 1.32$ & $0.046^{*}$ \\
$\Delta$ MMT & & & \\
\hline Hip & $0.50 \pm 0.66$ & $0.30 \pm 055$ & 0.173 \\
Knee & $0.57 \pm 0.67$ & $0.45 \pm 0.68$ & 0.225 \\
\hline Ankle & $0.10 \pm 0.75$ & $0.12 \pm 0.43$ & 0.525 \\
$\Delta$ K-MBI & $17.75 \pm 5.30$ & $13.25 \pm 7.65$ & 0.270 \\
$\Delta$ K-MMSE & $5.80 \pm 2.25$ & $4.95 \pm 2.44$ & 0.775 \\
\hline
\end{tabular}

Values are numbers or mean \pm standard deviation.

BBS, Berg Balance Scale; AP, anterior-posterior; ML, medial-lateral; MMT, manual muscle test; K-MBI, Korean version of Modified Barthel Index; K-MMSE, Korean version of Mini-Mental State Examination.

${ }^{*} \mathrm{p}<0.05$ by Mann-Whitney U-test.

Exercise therapy, the basis of rehabilitation therapy for improving standing ability, is a conventional exercisebased rehabilitation therapy administered under the supervision of a physical therapist. This type of therapy is usually applied as a primary measure because it is highly effective in improving the patient's standing ability [28]. However, it can only be performed by therapists and the exercise needs to be performed for only 30 minutes twice a day; therefore, additional exercise therapies that can be performed independently and in rest of the time by the patients are necessitated [29]. Exercise therapies that can be performed independently by the patients are being studied, and many rehabilitation devices for improving standing ability are constantly being used [30]. Bagley et al. [12] and Hendrie et al. [13] demonstrated that the standing frame is effective for improving the standing ability of stroke patients among other therapies. Matjacic et al. [30] also showed that the modified standing frame exercise is effective for improving the balance, mobility, independence, and functional ambulation in stroke patients. Also, the study emphasized that the modified standing frame exercise allows patients to exercise without the supervision of therapists, thereby increasing the patient's exercise time [31].

However, Nadeau et al. [14] reported that in clinical practice, most of the stroke patients tended to stand using only the healthy side without weight bearing on the hemiplegic side, and De Nunzio et al. [18] reported that the effectiveness of rehabilitation exercise for improving 
standing ability was enhanced in stroke patients when the problem of using only the healthy side during rehabilitation exercise was resolved. Therefore, various strategies for inducing weight bearing on the hemiplegic side have been proposed and studied. In a study by Bishop et al. [32], a tool to forcefully induce weight bearing on the hemiplegic side was developed using a pelvic assist device, and effectiveness was proven based on the performance of the device in an actual scenario. In the reported study, the authors reported improvement in functions when the patients performed exercise on their own without any help from the therapist. However, it was pointed out that it would be challenging to use this tool in clinical practice because of the cost and size of the device [32].

Therefore, the present study aimed to identify a method that would enable patients to exercise independently using only an instrument, thus solving the issue of inducing weight bearing only on the hemiplegic side, and be easily applicable in clinical practice. Among the various available methods that can increase the weight load on the hemiplegic side when patients perform the exercises independently, FES has recently been used in several studies. Bustamante et al. [22] reported that in addition to the previously recognized effectiveness of FES therapy in improving muscle strength of the leg on the hemiplegic side, it facilitated the perceptive development on the hemiplegic side by enhancing both the feedback of the sensory nerve and proprioceptive senses, ultimately increasing the use of the hemiplegic side. In addition, Chen et al. [33] demonstrated that performing standing rehabilitation exercise and gait rehabilitation exercise while wearing a device for FES therapy not only resulted in better functional improvement but also induced more activity than conventional rehabilitation therapy, as detected on brain diffusion tensor image. Previous studies $[34,35]$ demonstrated that a single session of FES-assisted treadmill exercise alone could induce an increase in the corticomotor plasticity. Furthermore, van Bloemendaal et al. [36] reported that the study group who underwent gait training along with FES showed higher functional improvement than the control group who underwent FES alone. The authors also demonstrated that FES-mediated brain stimulation in the study group subjects who received the combination therapy induced a synergistic effect with a resultant increase in brain plasticity. The reported studies demonstrated that FES therapy enhanced brain plasticity by increasing brain stimulation through sensory feedback, consequently increasing the perception and activity on the hemiplegic side and resulting in weight bearing and functional improvement. Ferrante et al. [37] showed that in addition to the reported effects, FES with volitional muscle contractions induced a synergistic effect, thus demonstrating the effectiveness of the combination therapy in improving the walking and standing ability than individual therapy.

In the present study, we hypothesized that the therapeutic effects of FES and standing frame can be combined to solve the problem of the existing rehabilitation therapy and aimed to prove this hypothesis.

Consequently, in this study, FES was attached to the anterior tibialis as it was contemplated that the peroneal nerve was stimulated to achieve the weight bearing effect on the hemiplegic side in response to sensory biofeedback, which was previously studied $[34,38]$. Similarly to conventional FES treatment, FES was attached to quadriceps femoris to treat knee collapse.

The results showed that standing balance training was more effective in the study group than in the control group, thus suggesting that the combination therapy has a high therapeutic effect and can solve the existing problem faced in standing frame training.

We considered three possible reasons for combination rehabilitation therapy being more effective than individual rehabilitation therapy. First, as noted in several studies, this study suggests that sensory biofeedback through FES may have increased brain stimulation and that this mechanism induced weight bearing on hemiplegia side during standing frame exercise. Therefore, we thought that this mechanism improved brain plasticity and improved standing balance ability. Second, the application of FES at the time of volitional muscle contractions during standing frame exercise induced a synergistic effect that is believed to contribute to enhanced improvement in the standing ability in the combination therapy group compared to the control group who received individual therapies. Third, 40 minutes of rehabilitation therapy time was given to the combination therapy group per session, while 80 minutes were given to the control group per session, thus imparting more time to the combination therapy group for other personal treatments.

To the best of our knowledge, this is the first study that attempted to solve the problems associated with standing 
frame training in stroke patients by applying FES. This study proved that combination rehabilitation therapy has additional therapeutic effects.

This study has some limitations, which have to be pointed out. First, as the therapeutic effect of combination therapy was evaluated only after a short-term exercise therapy period of three weeks, the long-term therapeutic effects should be evaluated in future studies. Second, given that the comparative analysis was performed by involving a relatively smaller number of patients, it will be necessary to conduct further research by employing a larger sample size in future studies.

In conclusion, the results demonstrate the therapeutic effectiveness of combined therapy of FES and standing frame in subacute stroke patients. The protocol is proposed to be time-saving and could be easily applied in the clinical setting. Accordingly, combined therapy could be a useful method to improve standing balance in subacute stroke patients.

\section{CONFLICT OF INTEREST}

No potential conflict of interest relevant to this article was reported.

\section{ACKNOWLEDGMENTS}

This study was carried out with a donation of rehabilitation robot in 2013 from the Ministry of Health and Welfare and National Rehabilitation Center as a part of robot supply business.

\section{REFERENCES}

1. Aze O, Odjardias E, Devillard X, Akplogan B, Calmels P, Giraux P. Structural and physiological muscle changes after post-stroke hemiplegia: a systematic review. Ann Phys Rehabil Med 2016;59 Suppl:e79.

2. Mohapatra S, Harrington R, Chan E, Dromerick AW, Breceda EY, Harris-Love M. Role of contralesional hemisphere in paretic arm reaching in patients with severe arm paresis due to stroke: a preliminary report. Neurosci Lett 2016;617:52-8.

3. Vistamehr A, Kautz SA, Bowden MG, Neptune RR. Correlations between measures of dynamic balance in individuals with post-stroke hemiparesis. J Biomech
2016;49:396-400.

4. de Haart M, Geurts AC, Huidekoper SC, Fasotti L, van Limbeek J. Recovery of standing balance in postacute stroke patients: a rehabilitation cohort study. Arch Phys Med Rehabil 2004;85:886-95.

5. Geurts AC, de Haart M, van Nes IJ, Duysens J. A review of standing balance recovery from stroke. Gait Posture 2005;22:267-81.

6. Hokstad A, Indredavik B, Bernhardt J, Langhammer B, Gunnes M, Lundemo C, et al. Upright activity within the first week after stroke is associated with better functional outcome and health-related quality of life: a Norwegian multi-site study. J Rehabil Med 2016;48: 280-6.

7. Cabanas-Valdes R, Bagur-Calafat C, Girabent-Farres M, Caballero-Gomez FM, Hernandez-Valino M, Urrutia Cuchi G. The effect of additional core stability exercises on improving dynamic sitting balance and trunk control for subacute stroke patients: a randomized controlled trial. Clin Rehabil 2016;30:1024-33.

8. Liu M, Chen J, Fan W, Mu J, Zhang J, Wang L, et al. Effects of modified sit-to-stand training on balance control in hemiplegic stroke patients: a randomized controlled trial. Clin Rehabil 2016;30:627-36.

9. Hugues A, Di Marco J, Janiaud P, Xue Y, Pires J, Khade$\mathrm{mi} \mathrm{H}$, et al. Efficiency of physical therapy on postural imbalance after stroke: study protocol for a systematic review and meta-analysis. BMJ Open 2017;7:e013348.

10. Beyaert C, Vasa R, Frykberg GE. Gait post-stroke: pathophysiology and rehabilitation strategies. Neurophysiol Clin 2015;45:335-55.

11. Zhang D, Zhu K. Simulation study of FES-assisted standing up with neural network control. Conf Proc IEEE Eng Med Biol Soc 2004;7:4877-80.

12. Bagley P, Hudson M, Forster A, Smith J, Young J. A randomized trial evaluation of the Oswestry Standing Frame for patients after stroke. Clin Rehabil 2005;19: 354-64.

13. Hendrie WA, Watson MJ, McArthur MA. A pilot mixed methods investigation of the use of Oswestry Standing Frames in the homes of nine people with severe multiple sclerosis. Disabil Rehabil 2015;37:1178-85.

14. Nadeau SM, Boukadida A, Piotte F, Mesure S. Weightbearing perception during standing and sit-to-stand tasks in subacute post-stroke individuals undergoing intensive rehabilitation. Ann Phys Rehabil Med 2016; 
59 Suppl:e71-e72.

15. Kim K, Kim YM, Kang DY. Repetitive sit-to-stand training with the step-foot position on the non-paretic side, and its effects on the balance and foot pressure of chronic stroke subjects. J Phys Ther Sci 2015;27: 2621-4.

16. Raja B, Neptune RR, Kautz SA. Quantifiable patterns of limb loading and unloading during hemiparetic gait: Relation to kinetic and kinematic parameters. J Rehabil Res Dev 2012;49:1293-304.

17. Montastruc J, Amarantini D, Lambert V, CastelLacanal E, Marque P, Gasq D. Main determinants of weight-bearing asymmetry in hemiplegic stroke patients. Ann Phys Rehabil Med 2016;59 Suppl:e69.

18. De Nunzio A, Zucchella C, Spicciato F, Tortola P, Vecchione C, Pierelli F, et al. Biofeedback rehabilitation of posture and weightbearing distribution in stroke: a center of foot pressure analysis. Funct Neurol 2014;29: 127-34.

19. Messier S, Bourbonnais D, Desrosiers J, Roy Y. Weightbearing on the lower limbs in a sitting position during bilateral movement of the upper limbs in post-stroke hemiparetic subjects. J Rehabil Med 2005;37:242-6.

20. Gervasoni E, Parelli R, Uszynski M, Crippa A, Marzegan A, Montesano A, et al. Effects of functional electrical stimulation on reducing falls and improving gait parameters in multiple sclerosis and stroke. PM R 2017;9:339-47.

21. Tan ZM, Jiang WW, Yan TB, Wu W, Song R. Effects of functional electrical stimulation based on normal gait pattern on walking function in subjects with recovery of stroke. Zhonghua Yi Xue Za Zhi 2016;96:2342-6.

22. Bustamante C, Brevis F, Canales S, Millon S, Pascual R. Effect of functional electrical stimulation on the proprioception, motor function of the paretic upper limb, and patient quality of life: a case report. J Hand Ther 2016;29:507-14.

23. Malesevic J, Strbac M, Isakovic M, Kojic V, Konstantinovic L, Vidakovic A, et al. Evolution of surface motor activation zones in hemiplegic patients during 20 sessions of FES therapy with multi-pad electrodes. Eur J Transl Myol 2016;26:6059.

24. Maeda N, Urabe Y, Murakami M, Itotani K, Kato J. Discriminant analysis for predictor of falls in stroke patients by using the Berg Balance Scale. Singapore Med J 2015;56:280-3.
25. Blum L, Korner-Bitensky N. Usefulness of the Berg Balance Scale in stroke rehabilitation: a systematic review. Phys Ther 2008;88:559-66.

26. Palm HG, Lang P, Strobel J, Riesner HJ, Friemert B. Computerized dynamic posturography: the influence of platform stability on postural control. Am J Phys Med Rehabil 2014;93:49-55.

27. Chen HX, Yang ZJ, Pan RH, Guo YH, Zhan LC, He MF, et al. Effect of comprehensive protocol of integrative medicine on motor function, activity of daily living and quality of life in hemiplegia patients after stroke. Zhongguo Zhong Xi Yi Jie He Za Zhi 2016;36:395-8.

28. Haruyama K, Kawakami M, Otsuka T. Effect of core stability training on trunk function, standing balance, and mobility in stroke patients. Neurorehabil Neural Repair 2017;31:240-9.

29. Grainger KP, Masterson S, Jennings M. 'Things aren't the same, are they?': the management of bad news delivery in the discourse of stroke care. Commun Med 2005;2:35-44.

30. Matjacic Z, Zadravec M, Oblak J. Sit-to-stand trainer: an apparatus for training "normal-like" sit to stand movement. IEEE Trans Neural Syst Rehabil Eng 2016; 24:639-49.

31. Braun T, Marks D, Thiel C, Zietz D, Zutter D, Gruneberg C. Effects of additional, dynamic supported standing practice on functional recovery in patients with sub-acute stroke: a randomized pilot and feasibility trial. Clin Rehabil 2016;30:374-82.

32. Bishop L, Khan M, Martelli D, Quinn L, Stein J, Agrawal S. Exploration of two training paradigms using forced induced weight shifting with the tethered pelvic assist device to reduce asymmetry in individuals after stroke: case reports. Am J Phys Med Rehabil 2017;96(10 Suppl 1):S135-40.

33. Chen D, Yan T, Li G, Li F, Liang Q. Functional electrical stimulation based on a working pattern influences function of lower extremity in subjects with early stroke and effects on diffusion tensor imaging: a randomized controlled trial. Zhonghua Yi Xue Za Zhi 2014;94:2886-92.

34. Thibaut A, Moissenet F, Di Perri C, Schreiber C, Remacle A, Kolanowski E, et al. Brain plasticity after implanted peroneal nerve electrical stimulation to improve gait in chronic stroke patients: Two case reports. NeuroRehabilitation 2017;40:251-8. 
35. Palmer JA, Hsiao H, Wright T, Binder-Macleod SA. Single session of functional electrical stimulationassisted walking produces corticomotor symmetry changes related to changes in poststroke walking mechanics. Phys Ther 2017;97:550-60.

36. van Bloemendaal M, Bus SA, de Boer CE, Nollet F, Geurts AC, Beelen A. Gait training assisted by multichannel functional electrical stimulation early after stroke: study protocol for a randomized controlled trial. Trials 2016;17:477.
37. Ferrante S, Chia Bejarano N, Ambrosini E, Nardone A, Turcato AM, Monticone M, et al. A personalized multi-channel FES controller based on muscle synergies to support gait rehabilitation after stroke. Front Neurosci 2016;10:425.

38. Khaslavskaia S, Sinkjaer T. Motor cortex excitability following repetitive electrical stimulation of the common peroneal nerve depends on the voluntary drive. Exp Brain Res 2005;162:497-502. 